\title{
Transcription factor AP-1 promotes growth and radioresistance in prostate cancer cells
}

\author{
RISTO KAJANNE $^{1,2}$, PÄIVI MIETTINEN ${ }^{3,4}$, MIKKO TENHUNEN $^{2}$ and SIRPA LEPPÄ ${ }^{1,2}$ \\ ${ }^{1}$ Molecular Cancer Biology Program, Biomedicum Helsinki, University of Helsinki; ${ }^{2}$ Department of Oncology, Helsinki \\ University Central Hospital; ${ }^{3}$ Hospital for Children and Adolescents, Helsinki University Central Hospital; ${ }^{4}$ Pediatric Research \\ Unit, HUCH Hospital for Children and Adolescents, Biomedicum Helsinki, University of Helsinki, Helsinki, Finland
}

Received May 14, 2009; Accepted July 10, 2009

DOI: 10.3892/ijo_00000434

\begin{abstract}
Expression of AP-1 proteins has been associated with a more aggressive clinical outcome in prostate cancer. However, their role and regulation by upstream kinase pathways in response to ionizing radiation has remained elusive. Here, we show that constitutive AP-1 activity in prostate cancer cells is dependent on the activities of EGF-R and PI3K. While inhibition of EGF-R is associated with suppression of c-Jun expression and proliferation, inhibition of PI3K pathway suppresses expression of several AP-1 subunits and proliferation, and also sensitizes prostate cancer cells to $\gamma$-radiation. The importance of AP-1 as a mediator of proliferation and radiation responses is demonstrated by the findings that the expression of JunD, Fra- 1 and Fra- 2 siRNAs in prostate cancer cells suppress these cellular responses. Together, the findings show that AP- 1 activity in prostate cancer cells mediates EGF-R and PI3K signalling, is essential for their proliferation, and confers protection against radiationinduced cell death. Thus, its inhibition would be a lucrative target for therapy in this widely increasing cancer type.
\end{abstract}

\section{Introduction}

Radiotherapy is the basis in managing early-stage or inoperable locally advanced prostate cancer. Recent innovations in three-dimensional conformal and intensity-modulated radiation therapy have allowed for maximal delivery of radiation to target volume while limiting toxicity $(1,2)$. Finding agents that sensitize malignant cells to radiation would further increase tumor response and minimize toxicity to surrounding organs, as effective therapeutic doses could be lowered.

Correspondence to: Dr Sirpa Leppä, Department of Oncology, P.O. Box 180, FIN-00029 Helsinki University Central Hospital, Helsinki, Finland

E-mail: sirpa.leppa@helsinki.fi

Key words: AP-1, EGF-R, AKT, growth, radiation, prostate cancer
One emerging candidate in modulating the radiation responses in prostate cancer is signaling mediated through the epidermal growth factor receptor (EGF-R). Increased EGF-R expression is often seen in prostate cancer and is associated with poor prognosis $(3,4)$. The activation of EGF-R triggers a complex network of downstream cellular pathways, modulating different cellular responses (5). According to in vitro studies, the activation of EGF-R signaling contributes notably to cell proliferation, survival and invasion of prostate cells. Furthermore, the blocking of EGF-R signaling by a selective EGF-R inhibitor, such as gefitinib, leads to growth inhibition and apoptosis of prostate cancer cells (6-8). However, clinical data demonstrate that not all patients respond to the inhibitor, indicating the existence of intrinsic or de novo resistance to the drug, and activation of alternative signaling pathways $(9,10)$.

$\mathrm{PI} 3 \mathrm{~K} / \mathrm{Akt}$ pathway is one of the major pathways mediating EGF-R signalling, and also a mediator of resistance to EGF-R $(9,11)$. Its constitutive activity has been implicated in many human cancers, including the prostate (12). This EGF-Rindependent activity is caused by mutations in the negative regulator of this pathway, PTEN, especially in the more advanced and aggressive forms of the disease $(12,13)$. Consequently, many prostate cancers have high levels of Akt activity.

Recently, data have been emerging to show a link between constitutive Akt activity and resistance to radiation in several cancer types. In prostate cancer cells, for example, inhibition of the PI3K/Akt pathway causes a decrease in survival after radiation (14). Importantly, the ability of LY294002 to act as a radiosensitizer is due to inhibition of Akt, and not an effect of this drug on other proteins.

Despite the advances in studying EGF-R- and PI3Kmediated signalling in prostate cancer cells, less information is available on the nuclear target molecules that mediate the biological effects of the pathways. Putative mediators of EGF$\mathrm{R}$ signalling include signal inducible transcription factors, such as the activator protein-1 (AP-1). AP-1 is a dimeric protein complex composed of different Jun (c-Jun, JunB and JunD) and Fos (c-Fos, Fra-1, Fra-2 and FosB) subunits (15). Upon activation the Jun homo- or Jun/Fos heterodimers are formed followed by DNA-binding activity, which together with other elements regulate target gene expression. The MAPK-mediated phosphorylation of AP-1 further stimulates 
their activities (16). However, other regulatory pathways, such as PI3K are also involved $(17,18)$. Interestingly, expression of AP-1 proteins has been recently associated with a more aggressive clinical outcome in prostate cancer (19). In the present study, we sought for the molecular mechanisms behind this, and found that AP-1 is a mediator of EGF-R and PI3K signalling during prostate cancer cell growth and radioresistance.

\section{Materials and methods}

Cell culture. Prostate cancer cell lines PC-3 and DU-145 were purchased from the American Type Culture Collection (ATCC; Manassas, VA). PC-3 cells were cultured in a humidified $5 \% \mathrm{CO}_{2}$ atmosphere at $37^{\circ} \mathrm{C}$ in $\mathrm{F}-12$ medium supplemented with $7 \%$ fetal calf serum (FCS), $2 \mathrm{mM}$ glutamine, $100 \mathrm{U} / \mathrm{ml}$ penicillin and $100 \mu \mathrm{g} / \mathrm{ml}$ streptomycin. DU-145 cells were grown in RPMI-1640 supplemented with $10 \%$ FCS $2 \mathrm{mM}$ glutamine, $100 \mathrm{U} / \mathrm{ml}$ penicillin and $100 \mu \mathrm{g} / \mathrm{ml}$ streptomycin. Chemical inhibitors for EGF-R [ZD1839 (gefinitib) used at $10 \mu \mathrm{M}$; Astra Zeneca, Cheshire, UK], PI3K (LY294002, used at 10 or $25 \mu \mathrm{M}$; Calbiochem, La Jolla, CA) and MEK (PD98059, used at $20 \mu \mathrm{M}$; Calbiochem) were added $12 \mathrm{~h}$ prior to radiation treatment.

Irradiation and colony forming assays. For clonogenic survival assays, cells were plated and allowed to attach overnight. The next day, cells were irradiated with a linear accelerator to a total of dose of 2, 4, or $8 \mathrm{~Gy}$. The irradiation was performed with the Varian Clinac 600C/D linear accelerator (Varian Medical Systems Inc.; Palo Alto, CA) using a 6-MV photon beam, and the dose rate of $4 \mathrm{~Gy} / \mathrm{min}$. Six hours after irradiation, cells were trypsinized, replated onto $3 \mathrm{~cm}$ dishes, and allowed to grow. In the experiments testing the radiosensitizing effects of EGF-R, MEK and PI3 kinase inhibitors, inhibitor treatment was initiated $12 \mathrm{~h}$ before irradiation, and stopped before replating. After 8 days, colonies were fixed with $2 \%$ PFA, stained with $0.5 \%$ crystal violet in ethanol and counted ( $>20$ cells). The statistical significance of differences seen in proliferation assays was analyzed using student's t-test. All p-values were two-tailed.

Analysis of proliferation. The cells were plated and allowed to grow overnight. The next day, they were trypsinized, replated onto $3 \mathrm{~cm}$ dishes in triplicates, and allowed to form colonies. In the experiments testing the short-term antiproliferative effects of EGF-R, MEK and PI3K inhibitors, inhibitor treatment was initiated after cell attachment, and stopped before replating, whereas in experiments testing the long-term effects of kinase inhibitors, treatment was reinitiated after efficient attachment of cells, and continued throughout the experiment. After 8 days, the samples were processed as described above. Alternatively, the cells were plated at a density of 50,000 cells/60-mm diameter well in duplicates and cultured for 3-7 days. In the experiments testing the growth inhibitory effect of kinase inhibitors, inhibitor treatment was initiated after efficient attachment of cells, and continued throughout the experiment. Cell number was determined after trypsinization using Burker chamber and light microscopy.
Analysis of cell-cycle arrest and apoptosis. For cell cyclearrest assays, cells were cultured for $24 \mathrm{~h}$ after irradiation, harvested and stained with propidium iodine in a hypotonic buffer. DNA fluorescence was analyzed by FACS (CellCalibur; Becton-Dickinson, Bedford, MA), and G1, S and G2 DNA fractions quantitated with ModFit software (Verity Software House Inc., Topsham, ME). To quantify apoptosis, portion of cells was harvested for Annexin V/propidium iodide assay according to the manufacturer's recommendation (APO-AF kit; Sigma, St. Louis, MO), and after 10 min analyzed using FACS and CellQuest software (BectonDickinson). The statistical significance of differences seen in proliferation and apoptosis assays was analyzed using student's t-test. All p-values were two tailed.

Western analysis. PC-3 and DU-145 cells were lysed into sodium dodecyl sulfate (SDS) sample buffer and sonicated. Equal amounts of proteins were separated on a $10 \%$ SDSpolyacrylamide gel and transferred onto nitrocellulose filters by electroblotting. Immunoblotting was performed using antibodies against phospho-EGF-R (44-974G; Invitrogen Corporation, Carlsbad, CA), EGF-R (E-3138; Sigma), phospho-Akt, (9272; Cell Signaling Technology, Beverly, MA), phospho-ERK (V803A; Promega, Madison, WI), ERK1/2 (sc-154; Santa Cruz Biotechnology Inc., Santa Cruz, CA), AP-1 proteins c-Jun (sc-45), JunB (sc-73), JunD (sc-74), c-Fos (sc-52), Fra-1 (sc-605), Fra-2 (sc-604) (all from Santa Cruz Biotechnology Inc.), as previously reported (20).

Gel mobility-shift assay. The cells were harvested and DNAbinding activity analyzed using oligonucleotide probe containing the 'consensus' AP-1 site as previously described (21).

Plasmids, siRNAs, transfections and luciferase assays. For transactivation studies, duplicates of $60-\mathrm{mm}$ diameter plates containing 50,000 cells were transfected with Luciferase reporter constructs for AP-1 (AP-1-Luc; Clontech, Mountain View, CA) using Lipofectamine 2000 reagent (Invitrogen). After $24 \mathrm{~h}$ the cells were collected and analyzed with Luciferase assay according to manufacturer's instructions (Promega). The activity of AP-1 reporter was normalized to the protein concentration.

For siRNA studies, PC-3 cells were transfected with $c$ Jun, Fra-2 (Proligo, Sigma), JunD, Fra-1, or siCONTROL (Dharmacon, Lafayette, CO) siRNAs (33 nmol) using Lipofectamin 2000 transfection reagent according to manufacturer's instructions (Invitrogen). Twenty-four hours after transfection, the cells were exposed to $\gamma$-irradation, and/or processed for further analyses.

\section{Results}

Radiosensitivity and proliferation of prostate cancer cells. To address cellular and molecular effects of EGF-R and their dependence on PI3K and MAPK pathway in prostate cancer, we performed experiments in PC-3 (PTEN-negative) and DU-145 (PTEN-positive) prostate cancer cell lines. Initially, PC-3 and DU-145 cells were exposed to specific EGF-R, $\mathrm{PI} 3 \mathrm{~K}$ and MEK inhibitors, and analyzed for proliferation and 
A.

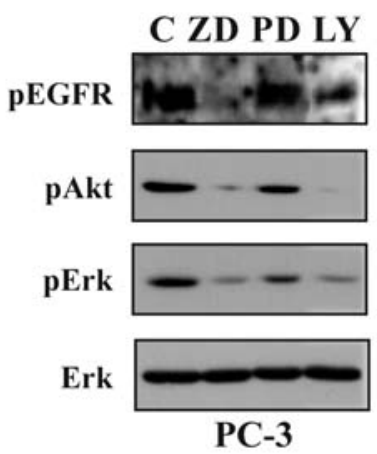

B.

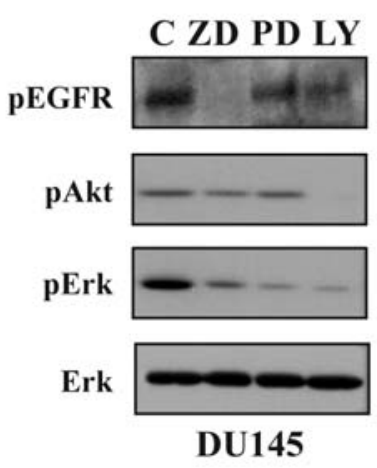

C.

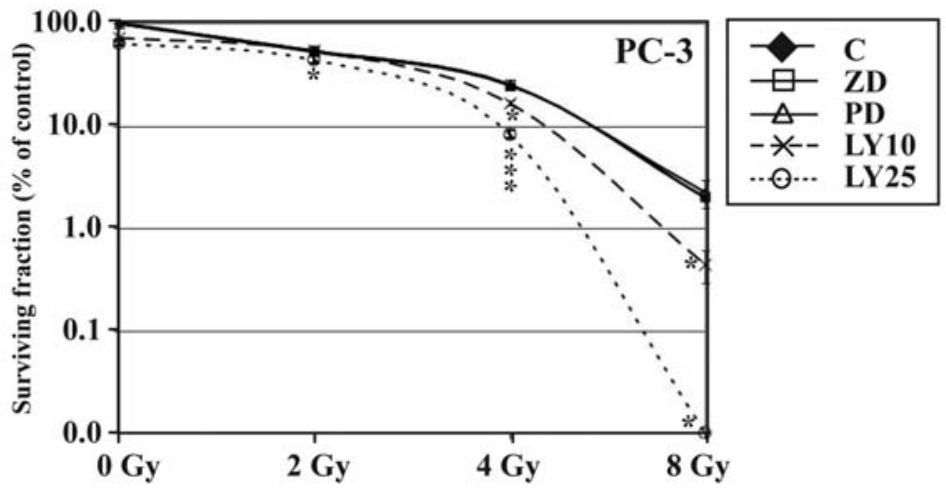

D.

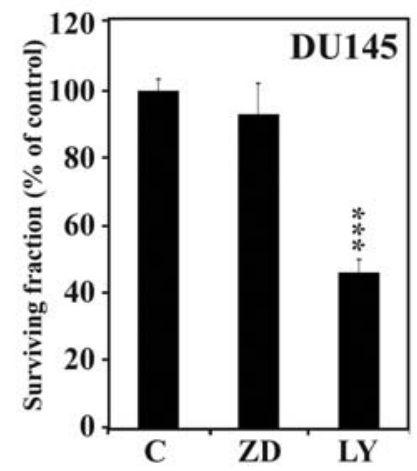

Figure 1. Inhibition of PI3K activity sensitizes PC-3 and DU-145 cells to ionizing irradiation. (A) and (B) Activity of EGF-R, Erk and PI3K pathways in PC-3 (A) and DU-145 cells (B) grown in the presence of EGF-R, Mek/Erk and PI3K/Akt inhibitors. PC-3 cells were incubated with kinase inhibitors specific for EGF-R (ZD) Mek/Erk (PD) and PI3K/Akt (LY) pathways for $24 \mathrm{~h}$. Protein lysates were prepared and total proteins (75 $\mu$ g) subjected to SDS-PAGE and immunoblotting using antibodies against phosphorylated EGF-R, Akt and Erk1/2. Total ERK1/2 was used as a loading control. (C) Clonogenic survival of the PC-3 cells. Untreated, control (C) or kinase inhibitor (ZD specific for EGF-R, PD for MEK, and LY for PI3K) pretreated PC-3 cells were exposed to radiation $(2,4$, or $8 \mathrm{~Gy})$. After $6 \mathrm{~h}$, equal amount of cells were plated onto 6-well plates for clonogenic survival assay. After 8 days cell colonies were fixed, stained with crystal violet and counted. Data are the mean \pm SD of three parallel samples. Statistically significant differences from values for control cells are indicated as follows: *, p <0.05; **, p <0.01; ***, p<0.005. Note that ZD and PD treated PC-3 samples overlay completely the controls. (D) Clonogenic survival of DU-145 cells. Untreated, control (C) or kinase inhibitor (ZD specific for EGF-R, PD for MEK, and LY for PI3K) pretreated DU-145 cells were exposed to radiation ( $4 \mathrm{~Gy}$ ), and processed for clonogenic survival assay as in panel C. Data are the mean \pm standard errors of three separate experiments. Statistically significant differences from values for controls $(C)$ are indicated with $* * *, p<0.005$.

radiation responses. The function of the inhibitors was confirmed on immunoblots using antibodies specific for the phosphorylated forms of EGF-R on tyrosine 1073, ERK and Akt (Figs. 1A and B). The EGF-R inhibitor (ZD) partially prevented ERK and Akt phosphorylation, whereas MEK inhibitor (PD) specifically suppressed ERK activity. PI3K inhibitor (LY) in turn, not only fully prevented Akt phosphorylation, but was also able to suppress EGF-R and ERK phosphorylation. This suppression is likely to be caused by inhibition of mTOR mediated global protein synthesis (22).

To address the effects of inhibitors on radiation-induced cell killing, PC-3 cells were pretreated with inhibitors for $12 \mathrm{~h}$, irradiated with 2, 4, or 8 Gy absorbed dose and after $6 \mathrm{~h}$ plated for colony forming assay in the absence of inhibitors (Fig. 1C). Consistent with studies in LNCaP prostate cancer cells (14), exposure to PI3K inhibitor (LY) sensitized PC-3 cells to radiation in a dose-dependent manner. In contrast, exposure to EGF-R inhibitor (ZD) neither protected nor sensitized PC-3 cells to radiation. The lack of EGF-R-inhibitor response was not due to lack of PTEN activity resulting in constitutively active PI3K/Akt pathway, since similar results were obtained with DU-145 cells, which have intact PTEN (Fig. 1D). Furthermore, exposure of PC-3 or DU-145 cells to EGF-R inhibitor (ZD) had no effect on colony formation.

Next, we analyzed the short- and long-term effects of the inhibitors on proliferation in the absence of radiation. PC-3 cells were pretreated with inhibitors for $18 \mathrm{~h}$, and plated for colony forming assay in the absence (short-term exposure) or in the presence (continuous treatment) of inhibitors. As shown in Fig. 2A, EGF-R inhibitor, which caused no growth inhibition after short exposure, precipitated $60 \%$ decrease in colony formation when followed for 8 days in culture. The anti-proliferative effects of PI3K inhibitor were already observed after short exposure and were further enhanced 
A.

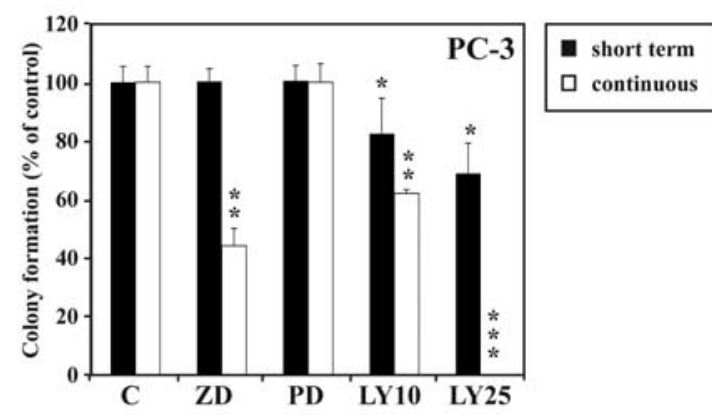

C.

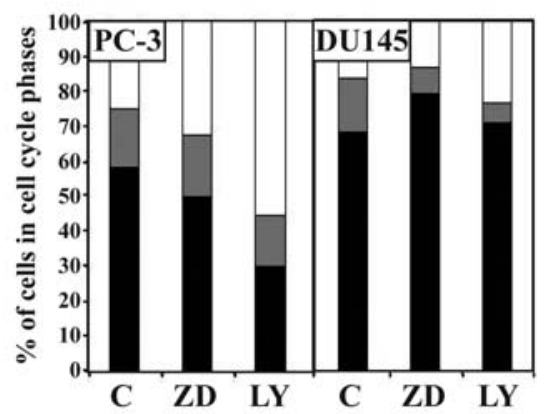

B.

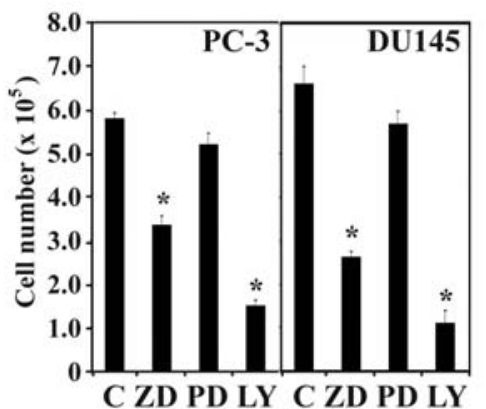

D.

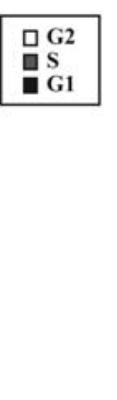

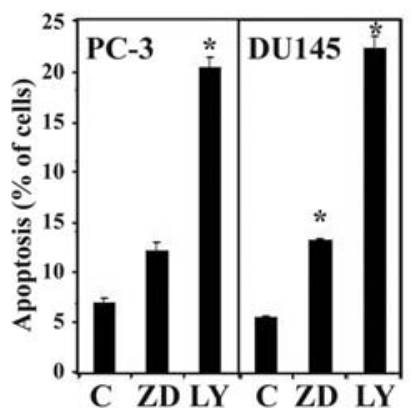

Figure 2. Inhibition of EGF-R or PI3K affects growth, cell-cycle distribution and apoptosis of prostate cancer cells. (A) Clonogenic survival of PC-3 in the presence of kinase inhibitors. PC-3 cells were treated with kinase inhibitors specific for EGF-R (ZD), Mek/Erk (PD) and PI3K/Akt (LY) pathways for $18 \mathrm{~h}$ (short-term exposure) or throughout the experiment (continuous exposure), and after 8 days processed for clonogenic survival assay. Data are the mean \pm standard errors of three experiments with three parallel samples. Statistically significant differences from values for control cells are indicated as follows: $*, \mathrm{p}<0.05$; **, $\mathrm{p}<0.01$; ***, $<<0.005$. (B) Proliferation in the presence of kinase inhibitors. PC-3 and DU-145 cells were cultured in the presence of kinase inhibitors and after 5 days trypsinized and counted. Data are the mean \pm standard errors of two samples. Statistically significant differences from values for control cells are indicated with an asterisk $(\mathrm{p}<0.05)$. (C) Cell-cycle distribution in the presence of kinase inhibitors. Untreated $(\mathrm{C})$ or kinase inhibitor pretreated (ZD for EGF-R, PD for MEK, and LY for PI3K) PC-3 and DU-145 cells were exposed to radiation and after 48 h harvested into hypotonic propidium iodine-solution, and analyzed for cell-cycle distribution by FACS. (D) Apoptosis in the presence of kinase inhibitors. PC-3 and DU-145 cells were treated as in panel B but analyzed for apoptosis using Annexin V and propidium iodine stainings. Data are the mean \pm standard errors of two separate experiments. Statistically significant differences from values for controls $(C)$ are indicated with an asterisk $(\mathrm{p}<0.05)$.

with continuous treatment (Fig. 2A). Similar results were obtained when PC-3 or DU-145 cells were detached after the treatments and counted separately (Fig. 2B). Together with the findings in Figs. $1 \mathrm{C}$ and D, the results provide evidence that EGF-R activity is required for proliferation, whereas PI3K also regulates sensitivity to radiation independently of EGF-R function. In comparison, MEK inhibitor (PD) affected neither radiosensitivity nor proliferation, indicating that MAPK pathway was dispensable for either cell responses (Figs. 1C and D, and 2A).

Cell-cycle progression and apoptosis. To identify the molecular mechanism involved in radiosensitization, cellcycle distribution was analyzed. PC-3 and DU-145 cells were treated with inhibitors, irradiated and monitored over a period of $24 \mathrm{~h}$ by DNA staining with propidium iodide and FACS analysis. As shown in Fig. 2C, radiation caused a G2 arrest with concomitant decrease of cells in G1 phase. When the cells were treated with the PI3K inhibitor, the number of cells arrested in G2 further increased. In contrast, no clear effect was noted either with the EGF-R or the MEK inhibitors. In the absence of radiation, neither EGF-R nor MEK inhibitors influenced the cell-cycle distribution, whereas treatment with the PI3K inhibitor led to a G1 arrest with a simultaneous decrease in cells in $\mathrm{G} 2$ and $\mathrm{S}$ phases.

The relative contribution of apoptosis to decreased cell growth and colony formation in response to the EGF-R and PI3K inhibitors and radiation was measured with an Annexin V flow cytometry apoptosis assay. Fig. 2D shows that apoptosis was not upregulated in response to radiation alone. The most prominent increase in apoptosis was obtained with the PI3K inhibitor both in the control and the irradiated cells. The EGF-R inhibitor doubled the apoptotic rate in the absence of radiation. However, no additive apoptotic effect was seen 
A. B.

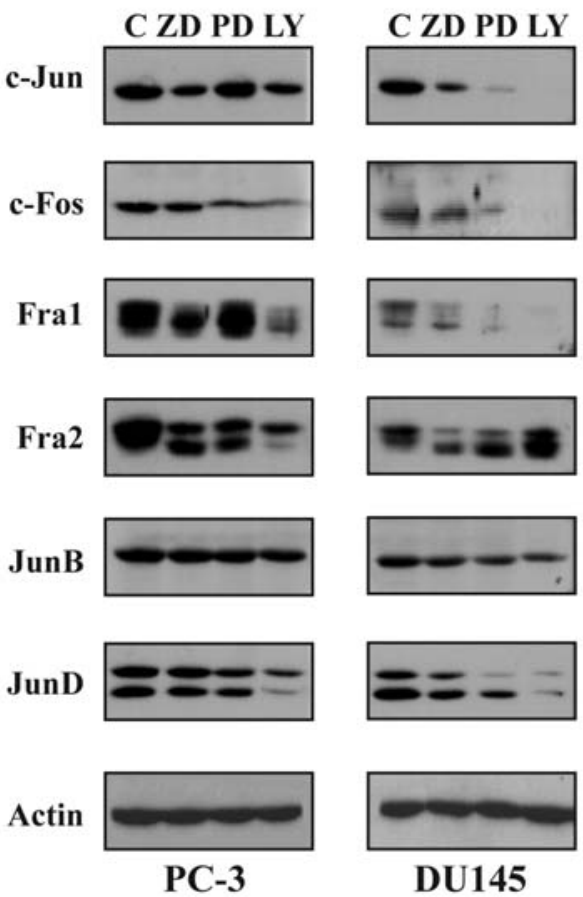

Figure 3. Expression of AP-1 proteins in prostate cancer cells grown in the presence of EGF-R, Mek/Erk and PI3K/Akt inhibitors. PC-3 (A) and DU145 (B) cells were incubated with kinase inhibitors specific for EGF-R (ZD), Mek/Erk (PD) and PI3K/Akt (LY) pathways for $24 \mathrm{~h}$. Protein lysates were prepared and total proteins $(75 \mu \mathrm{g})$ subjected to SDS-PAGE and immunoblotting using antibodies against Jun and Fos proteins. Actin was used as a loading control. after combining the PI3K or EGF-R inhibitor with radiation. The MEK inhibition had no effect on apoptosis.

Expression and activity of AP-1 subunits in prostate cancer cells. It is well established that EGF-R mediates signalling via PI3K and MAPK pathways and that the transcription factor AP-1 is the potential target to mediate the biological effects of these pathways $(5,11,17,18,20)$. To identify the dependence of AP-1 activity on EGF-R, PI3K and MAPK signalling, and its possible regulatory function in prostate cancer cell growth and radiosensitivity, the PC-3 and DU145 cells were cultured in the presence of EGF-R, PI3K and MEK inhibitors, and analyzed for the expression and activity of Jun and Fos proteins. As shown in Fig. 3, all Jun and Fos subunits were constitutively expressed both in the PC-3 and DU-145 cells. Blocking the PC-3 cells with the PI3K resulted in a decrease in the expression of all the other AP- 1 subunits except JunB. The levels of c-Fos and Fra-1 were most prominently suppressed. The MEK inhibitor in turn suppressed c-Fos levels strongly and Fra-2 levels only faintly. Likewise, the EGF-R inhibitor prevented the expression of c-Jun, Fra-1 and Fra-2 only slightly. The weak inhibitory effect of the EGF-R blockade was not caused by PTEN deficiency, since similar data was obtained in DU-145 cells, which have wildtype PTEN (Fig. 3B). The results suggest that the PI3K pathway functions as an essential regulator of Jun and Fos expression in the prostate cancer cells, whereas the role of EGF-R and MEK kinases might be less important.

To examine the AP-1 DNA-binding activity of the PC-3 and DU-145 cells, a gel mobility shift assay was performed. As shown in Fig. 4A, a strong constitutive AP-1 DNAbinding activity was observed in both cell lines. Consistent with the protein data in Fig. 3, the EGF-R and PI3K inhibitors
A.

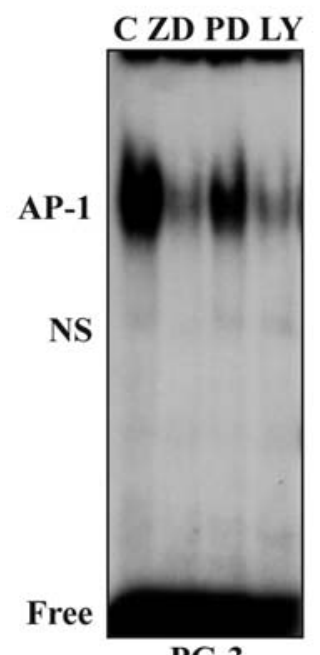

PC-3
B.

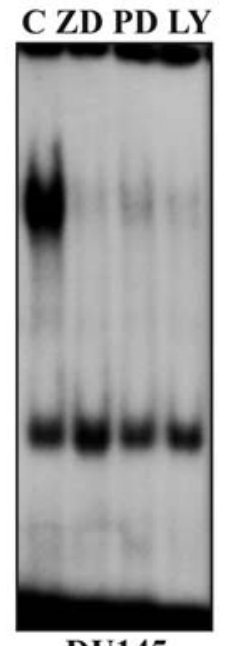

DU145

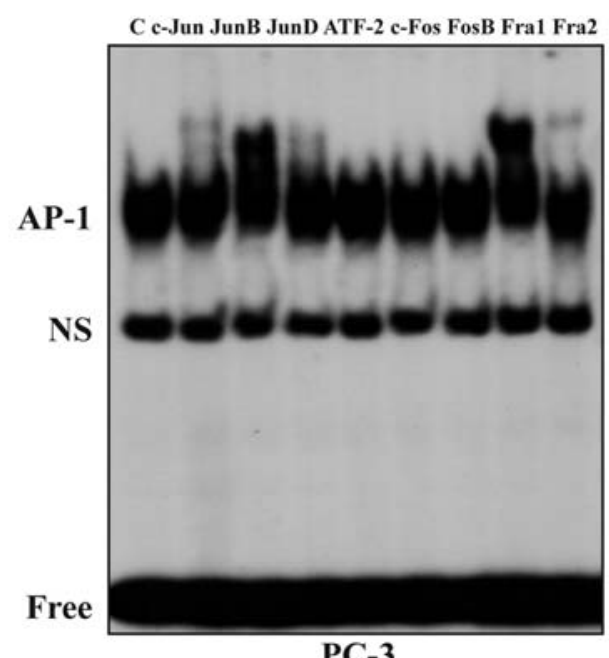

PC-3
C.
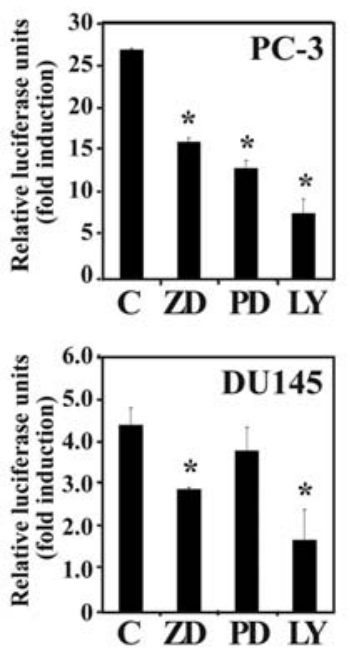

Figure 4. AP-1 DNA-binding and transactivation activities are dependent on EGF-R, Akt and Erk activities in PC-3 cells. (A) Analysis of AP-1 DNA-binding activity. Whole-cell extracts isolated from control and kinase inhibitor-treated PC-3 and DU-145 cells were analyzed by gel mobility shift assay using TRE oligonucleotide probe. AP-1 indicates the AP-1/TRE complex, NS indicates non-specific DNA-protein complexes, and free indicates unbound TRE oligonucleotides. (B) Analysis of AP-1 DNA-protein complex formation. Whole cell extracts isolated from PC-3 cells were pre-incubated with antibodies against AP-1 proteins prior to gel mobility shift assay. Supershifting bands indicate the slower migrating protein-antibody complexes. (C) Analysis of AP-1 transactivation capacity. PC-3 and DU-145 cells were transfected with AP-1 luciferase construct (AP-1-Luc) and treated with kinase inhibitors for 24 h. Cells were harvested and analyzed for firefly luciferase activities using luminometer. The luciferase activity was normalized against protein concentration. The data are the mean \pm standard errors of two parallel samples. Statistically significant differences from values for control are indicated with an asterisk ( $<<0.05$ ). 
A.
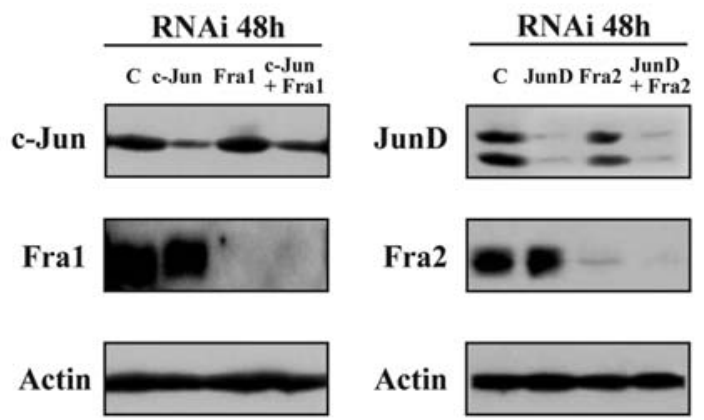

C.

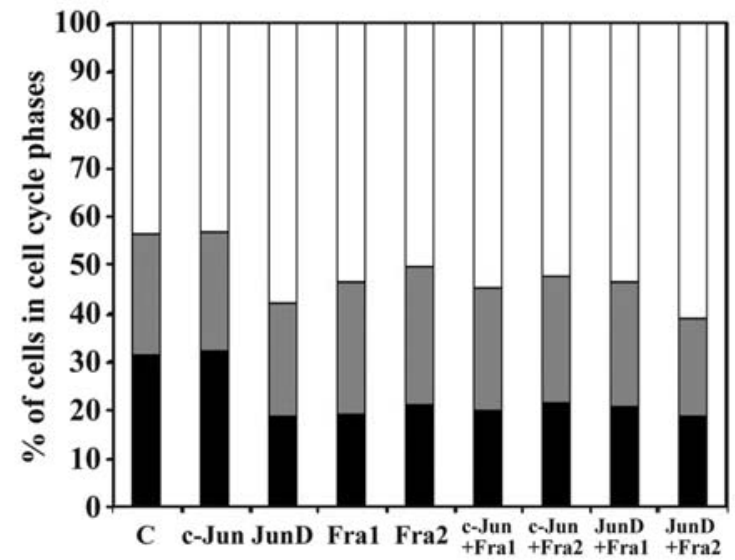

B.

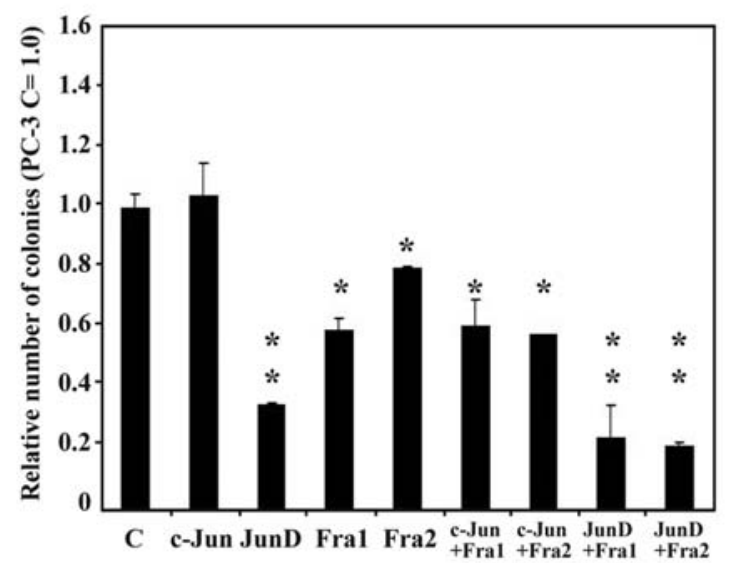

D.

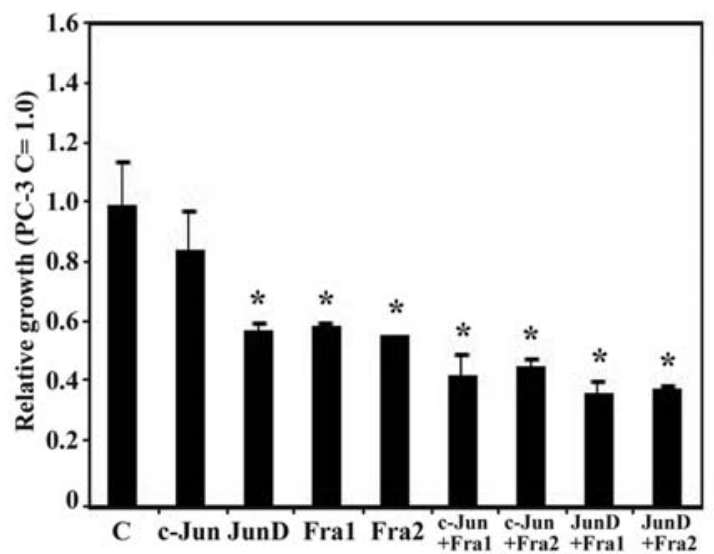

Figure 5. Downregulation of specific AP-1 components decreases growth and sensitizes prostate cancer cells to irradiation. (A) PC-3 cells were transfected with $c$-Jun, JunD, Fra-1, Fra-2, or control siRNAs, as indicated. After $48 \mathrm{~h}$ protein lysates were prepared and total proteins (75 $\mu \mathrm{g}$ ) subjected to SDS-PAGE and immunoblotting using antibodies against indicated AP-1 subunits. Actin was used as a loading control. (B) Clonogenic survival assay. PC-3 cells were transfected as in panel A and irradiated (4 Gy). Equal amount of cells were plated onto 6-well plates and cultured. After 8 days cell colonies were fixed, stained with crystal violet and counted. Data are the mean \pm S.D of three samples. Statistically significant differences from values for control cells are indicated as follows: *, p <0.05; **, $\mathrm{p}<0.01$; ***, $<<0.005$. (C) Cell-cycle analysis. PC-3 cells were processed as in panel A, and irradiated (4 Gy) but after $48 \mathrm{~h}$ harvested into hypotonic propidium iodine-solution and analyzed for cel-cycle distribution by FACS. The data are the mean \pm standard errors of two separate experiments. (D) Proliferation assay. PC-3 cells were transfected as in panel A. After 5 days the cells were trypsinized and counted. The data are the mean \pm standard errors of two parallel samples. Statistically significant differences from values for control cells are indicated with an asterisk ( $<<0.05)$.

prominently inhibited the AP-1 DNA-binding activity, while the MEK inhibitor had only a modest suppressive effect on DNA-binding.

The composition of the active AP-1 DNA-binding complex was determined using antibody perturbation assays. The PC-3 cell extracts were incubated with antibodies against the different Fos and Jun proteins and thereafter subjected to gel mobility shift analysis. Fig. 4B shows that Fra-1 and Fra-2 were the Fos-family subunits in the DNA-protein complex. All the Jun proteins were present in the DNA-binding complex, whereas antibodies against c-Fos, FosB and ATF-2 did not have any effect on complex formation. Similar results were obtained from the DU-145 cells.

To test whether the inhibitor-mediated decrease in AP-1 DNA-binding activity had functional consequences for the prostate cancer cells, we analyzed the AP-1 transactivation capacity. As shown in Fig. 4C, transcriptionally active AP-1 complexes were present in the normally proliferating $\mathrm{PC}-3$ and DU-145 cells, and all inhibitors were able to suppress the promoter activities in transfected cells. Again, the most prominent inhibition was observed with the PI3K blockade.

Fra-1, Fra-2 and JunD subunits are required for proliferation and resistance to radiation. Having demonstrated that AP-1 is constitutively active, and regulated particularly by PI3K, we assessed the function of AP-1 subunits in the PC-3 cells separately. According to the data described above, the most likely AP-1 components mediating PI3K-dependent cellular responses were Fra-1, Fra-2 and JunD. To test their functional role in the PC-3 cells, we analyzed proliferation and radiosensitivity of the PC-3 cells after knocking down the expression of Fra-1, Fra-2 and JunD with siRNAs. Based on previous 
studies showing that deletion of c-Jun can prevent proliferation (23), c-Jun siRNA was chosen as the positive control, whereas SiCONTROL siRNA served as a negative control. The cells were transfected with siRNAs together with EGFP Spectrin, and analyzed for the expression of c-Jun, JunD, Fra- 1 and Fra- 2 proteins by immunoblotting. The analysis verified a marked downregulation of the corresponding AP-1 subunits in comparison to the PC-3 cells transfected with only the control siRNAs (Fig. 5A).

When the PC- 3 cells were irradiated with 4 Gy, approximately $25 \%$ of the cells survived. Transfection of JunD, Fra-1 and Fra-2 siRNAs into the PC-3 cells further sensitized them to irradiation by reducing the colony formation to 36,58 and $78 \%$, respectively, as compared to control (C) and c-Jun siRNAs (Fig. 5B). Combination of $c$-Jun siRNAs with Fra-1 or Fra-2 siRNAs did not further increase radiation-induced killing, in comparison to each siRNAs alone. In contrast, combination of JunD siRNA with Fra-1 or Fra-2 resulted in a greater reduction in colony formation than either siRNA alone. Concomitantly, the proportion of cells arrested in G2/M phase increased (Fig. $5 \mathrm{C}$ ). The results demonstrate that JunD, Fra-1 and Fra-2, but not c-Jun are necessary for optimal clonogenic survival of the PC-3 cells.

Transfection of JunD, Fra-1 and Fra-2 siRNAs into normally growing PC-3 cells also reduced their growth rate significantly (Fig. 5D). In contrast, $c$-Jun siRNA had no significant effect on the cell proliferation. This together with our previous studies indicates that JunD, Fra-1 and Fra-2 are regulated by the $\mathrm{PI} 3 \mathrm{~K}$ pathway and are essential for both the radioresistance and proliferation of $\mathrm{PC}-3$ cells.

\section{Discussion}

Here we have analyzed how the EGF-R, PI3K and MAPK/ ERK pathways regulate AP-1 subunits during proliferation and radiation response of $\mathrm{PC}-3$ and DU-145 prostate cancer cells. Our results show that both responses require PI3K signalling, which culminates to c-Fos, Fra-1, Fra-2, JunD and c-Jun expression. Of these, c-Fos is not involved in the active DNA-binding complex. We further provide evidence that the PI3K-dependent activation of Fra-1, Fra-2 and JunD is indispensable for radioresistance and growth of PC-3 cells, whereas C-Jun is not required for these responses. The capacity of the AP-1 subunits to promote proliferation and radioresistance is most prominent when JunD is co-expressed with Fra-1 or Fra-2. In comparison to the PI3K pathway, the EGF-R activity is required only for cell proliferation, and MAPK/ERK pathway does not appear to be essential for either response.

The major mediators of the EGF-R-dependent signals are the PI3K/Akt and MAPK/ERK pathways $(5,24)$. Because we could suppress the ERK activity without altering cell proliferation and clonogenic survival, our data suggest that the MAPK/ERK pathway plays no role in the growth response of PC-3 or DU-145 cells. Thus, although the EGF-R activity is required for growth, the signaling downstream of EGF-R does not proceed via the conventional mitogenic MEK/ERK kinase cascade. Considering our finding that the PI3K inhibitor suppressed growth and the fact that only a low percentage of the cells underwent active proliferation, the pathways regulating survival (PI3K/Akt pathway) rather than proliferation (MAPK/ERK pathway) are likely to be the key components mediating prostate cancer growth.

Our results further confirm previous observations linking activated Akt with radioresistance of prostate cancer (14). While blockade of the PI3K decreased Akt activation and sensitized the PC-3 and DU-145 cells to radiation, no such effect was seen with the EGF-R and MEK inhibitors, indicating that the PI3K/Akt function is specific and critical for the radiation response of the PC-3 cells. The inability of EGF-R to confer radioresistance further indicates that radioresistance and proliferation are uncoupled in these cells. However, the data also demonstrate that molecular mechanisms mediating this radioresistance cannot be exclusively explained by defective PTEN function resulting in constitutively active Akt pathway, since the PI3K inhibitor-dependent radiosensitization was also observed in the DU-145 cells, which have no mutations in the PTEN gene. Additional studies are required to determine the Akt-dependent and PTEN-independent mechanism of radioresistance. Because LY294002 is a nonselective PI3K inhibitor and has multiple targets (25), we cannot exclude the possibility that some of the observed effects are partially mediated through inhibition of other kinases. However, the ability of the constitutively active Akt to block LY294002-induced radiosensitization in prostate cancer cells (14) suggests that the decreased survival demonstrated with the PI3K inhibition is a direct consequence of the inactivation of Akt.

In contrast to the consolidated proof concerning Akt function in survival and radioresistance, little is known about the role of AP-1 in mediating these cellular responses. Thus far, the only compelling evidence that Fra-1 is regulated by Akt pathway has been obtained in the PC-3 cells by using a PI3K inhibitor and microarray analyses (18). Our findings are in agreement with the data and further suggest an essential role for Fra-1 as well as Fra-2 and JunD in the regulation of cell growth. Accordingly, the PC-3 cells, in which the expression of these AP-1 subunits was silenced, had impaired G2 progression. Interestingly, similar effects on the cell cycle distribution have recently been described in human breast and thyroid cancer cell lines $(26,27)$. The molecular mechanism mediating the radioprotective effects of Fra-1, Fra-2 and JunD is currently undefined, and additional studies are in progress to precisely define the mechanisms by which their suppression reverses resistance to radiation.

Taken together, our data show that the activation of Fra-1, Fra-2 and JunD contributes to prostate cancer growth and survival after radiation in a PI3K-dependent manner. In contrast, c-Jun activity is associated with neither response. The findings demonstrate that although the AP-1 activity is implicated in various cellular processes, it is not the activity itself but rather the composition of the subunits, which contributes to the final outcome.

\section{Acknowledgements}

We thank Dr Tuomo Alanko for critical reading of the manuscript. The study was supported by fellowships from the Finnish Academy of Sciences (S.L.), Finnish Cancer Societies (S.L.), Sigrid Juselius (S.L.), Paolo (R.K.), and K. Albin 
Johansson (R.K.) Foundations, University of Helsinki (S.L.) and Helsinki University Central Hospital (S.L.). Gefitinib $\left(\right.$ Iressa ${ }^{\circledR}$ ) was provided by AstraZeneca.

\section{References}

1. Pollack A, Hanlon AL, Horwitz EM, Feigenberg SJ, Uzzo RG and Hanks GE: Prostate cancer radiotherapy dose response: an update of the fox chase experience. J Urol 171: 1132-1136, 2004.

2. Zelefsky MJ, Fuks Z, Hunt M, et al: High dose radiation delivered by intensity modulated conformal radiotherapy improves the outcome of localized prostate cancer. J Urol 166 : 876-881, 2001

3. Di Lorenzo G, Tortora G, D'Armiento FP, et al: Expression of epidermal growth factor receptor correlates with disease relapse and progression to androgen-independence in human prostate cancer. Clin Cancer Res 8: 3438-3444, 2002.

4. Schlomm T, Kirstein P, Iwers L, et al: Clinical significance of epidermal growth factor receptor protein overexpression and gene copy number gains in prostate cancer. Clin Cancer Res 13: 6579-6584, 2007.

5. Yarden Y and Sliwkowski MX: Untangling the ErbB signalling network. Nat Rev Mol Cell Biol 2: 127-137, 2001.

6. Sgambato A, Camerini A, Faraglia B, et al: Targeted inhibition of the epidermal growth factor receptor-tyrosine kinase by ZD1839 ('Iressa') induces cell-cycle arrest and inhibits proliferation in prostate cancer cells. J Cell Physiol 201: 97-105, 2004.

7. Sirotnak FM, She Y, Lee F, Chen J and Scher HI: Studies with CWR22 xenografts in nude mice suggest that ZD1839 may have a role in the treatment of both androgen-dependent and androgen-independent human prostate cancer. Clin Cancer Res 8: 3870-3876, 2002.

8. Vicentini C, Festuccia C, Gravina GL, Angelucci A, Marronaro A and Bologna M: Prostate cancer cell proliferation is strongly reduced by the epidermal growth factor receptor tyrosine kinase inhibitor ZD1839 in vitro on human cell lines and primary cultures. J Cancer Res Clin Oncol 129: 165-174, 2003.

9. Camp ER, Summy J, Bauer TW, Liu W, Gallick GE and Ellis LM: Molecular mechanisms of resistance to therapies targeting the epidermal growth factor receptor. Clin Cancer Res 11: 397-405, 2005.

10. Ciardiello F and Tortora G: EGFR antagonists in cancer treatment N Engl J Med 358: 1160-1174, 2008.

11. Cantley LC: The phosphoinositide 3-kinase pathway. Science 296: 1655-1657, 2002

12. Majumder PK and Sellers WR: Akt-regulated pathways in prostate cancer. Oncogene 24: 7465-7474, 2005.
13. McMenamin ME, Soung P, Perera S, Kaplan I, Loda M and Sellers WR: Loss of PTEN expression in paraffin-embedded primary prostate cancer correlates with high Gleason score and advanced stage. Cancer Res 59: 4291-4296, 1999.

14. Gottschalk AR, Doan A, Nakamura JL, Stokoe D and HaasKogan DA: Inhibition of phosphatidylinositol-3-kinase causes increased sensitivity to radiation through a PKB-dependent mechanism. Int J Radiat Oncol Biol Phys 63: 1221-1227, 2005.

15. Angel P and Karin M: The role of Jun, Fos and the AP-1 complex in cell-proliferation and transformation. Biochim Biophys Acta 1072: 129-157, 1991.

16. Whitmarsh AJ and Davis RJ: Transcription factor AP-1 regulation by mitogen-activated protein kinase signal transduction pathways. J Mol Med 74: 589-607, 1996.

17. Cao H, Dronadula N and Rao GN: Thrombin induces expression of FGF-2 via activation of PI3K-Akt-Fra-1 signaling axis leading to DNA synthesis and motility in vascular smooth muscle cells. Am J Physiol Cell Physiol 290: C172-C182, 2006.

18. Tiwari G, Sakaue H, Pollack JR and Roth RA: Gene expression profiling in prostate cancer cells with Akt activation reveals Fra-1 as an Akt-inducible gene. Mol Cancer Res 1: 475-484, 2003.

19. Ouyang X, Jessen WJ, Al-Ahmadie H, et al: Activator protein-1 transcription factors are associated with progression and recurrence of prostate cancer. Cancer Res 68: 2132-2144, 2008.

20. Kajanne R, Miettinen P, Mehlem A, et al: EGF-R regulates MMP function in fibroblasts through MAPK and AP-1 pathways. J Cell Physiol 212: 489-497, 2007.

21. Eriksson M and Leppa S: Mitogen-activated protein kinases and activator protein 1 are required for proliferation and cardiomyocyte differentiation of P19 embryonal carcinoma cells. J Biol Chem 277: 15992-16001, 2002.

22. Brunn GJ, Hudson CC, Sekulic A, et al: Phosphorylation of the translational repressor PHAS-I by the mammalian target of rapamycin. Science 277: 99-101, 1997.

23. Schreiber M, Kolbus A, Piu F, et al: Control of cell cycle progression by c-Jun is p53 dependent. Genes Dev 13: 607-619, 1999.

24. Prenzel N, Fischer OM, Streit S, Hart S and Ullrich A: The epidermal growth factor receptor family as a central element for cellular signal transduction and diversification. Endocr Relat Cancer 8: 11-31, 2001

25. Stein RC: Prospects for phosphoinositide 3-kinase inhibition as a cancer treatment. Endocr Relat Cancer 8: 237-248, 2001.

26. Belguise K, Kersual N, Galtier F and Chalbos D: FRA-1 expression level regulates proliferation and invasiveness of breast cancer cells. Oncogene 24: 1434-1444, 2005.

27. Casalino L, Bakiri L, Talotta F, et al: Fra-1 promotes growth and survival in RAS-transformed thyroid cells by controlling cyclin A transcription. EMBO J 26: 1878-1890, 2007. 